\title{
Controllable Motion Textures
}

\author{
Tse-Hsien Wang* Chun-Tse Hsiao* Bing-Yu Chen ${ }^{*} \quad$ Pei-Zhi Huang ${ }^{\dagger}$ \\ *National Taiwan University 部he University of Tokyo 'Digimax
}

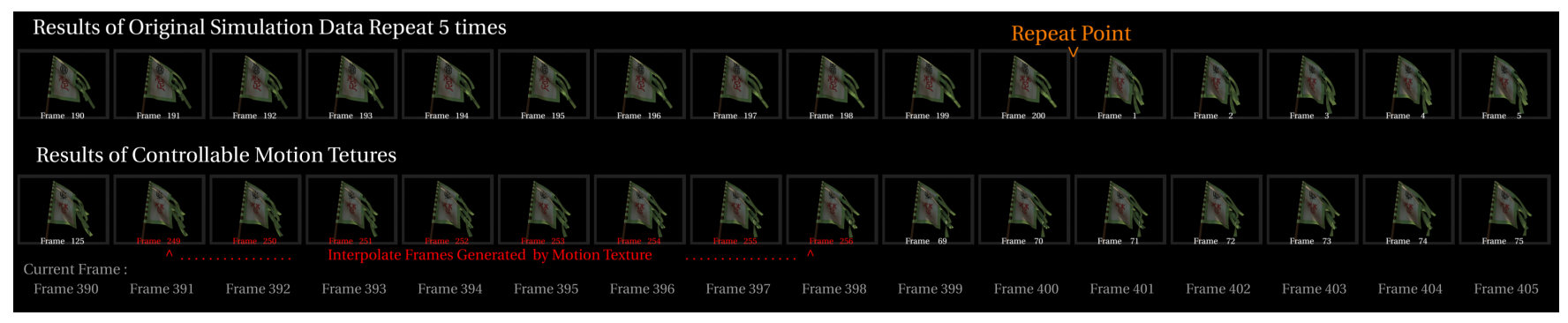

\section{Introduction}

While producing an animation, we often rely on the artist to make it manually, or sometimes we generate the animation by some physical simulations. However, the animation we created by these methods always has fixed length and is very difficult to reuse. For 3D animation production, in practice, we always need some animations with variable lengths, for example, we would like to place some flags waving in the wind as part of the background, also perhaps nearby some trees standing with their swaying branches. It will be significantly time-consuming and inefficient to make fixed-length animations (flags and trees) for a specified scene every time when the scene is altered. Consequently, suppose we have produced a segment of an animation, then based on some analysis of it, we could randomly generating a new animation, which length is specified as user's pleases. For example, we could generate an animation which shows a square surrounding by tens of flags swinging differently by using only one short flag animation originally. By doing this, the time for making films could considerably be saved. In this paper, we transform the input animation into a medium called motion texture, which could be used to generate random-length animations. A motion texture records lots of parts of an animation and the relations between these segments. According to the information, we could generate a large amount of different animations we require in the future.

\section{Algorithm}

\subsection{Defining the Cost}

First of all, we have to identify the cost between every two frames in the animation. We could think each frame as a 3D model for simplicity. There are three aspects we used to compute the cost: the distance, the velocity and the acceleration of each corresponding vertex. We derive unit velocity vectors of each paired vertices in two frames and define the cost function between the frames $i$ and $j$ as:

$$
\max _{v \in \mathcal{V}}\left\|V_{i, v}-V_{j, v}\right\|^{2}
$$

The heuristic is that we prefer the two frames have the same velocity orientations such that we can easily fill the gap with cubic spline interpolation. Furthermore, we assign $\infty$ cost if more than $20 \%$ of velocity vectors in frame $i$ is different then morphing direction which derived by vertex position to avoid sharp invert motions.

\footnotetext{
*e-mail: \{starshine,hsiaochm,robin\}@ cmlab.csie.ntu.edu.tw

†e-mail: ttyellow@ms.digimax.com.tw
}

\subsection{Making the Transitions}

Once the frame-to-frame costs have been calculated, the transition locations should be determined. The frames having less costs will be taken into consideration first. However, to avoid monotonous result caused by concentrated transition locations, we use some heuristics to balance the costs and the distances between these transition locations. Then, while we play the animation normally, at each transition location it may have several choices, we look up the information in the motion texture data structure and randomly choose a frame to jump to. Sometimes we could not obtain enough transitions which have low costs, as a result we must use morphing technique to make the transitions as smooth as possible. The morphing frames are generated by cubic spline interpolation and the frame number of it depends on the cost distance between the two transition frames.

\section{Result and Conclusion}

The result is shown in our demo video and displayed as the teaser in this paper. Our motion texture system could save large amount of time for 3D scene production. Though it works best for simple 3D models with smooth motion now, it still making the production of animation easier and faster. Although in our implementation, the cost function and morphing scheme cannot capture spatial regular motion pattern such as wavy motion of flags, so we can easily observe some strange smooth transition of flag synthesized animation in our demo video.

There are some techniques which could make the motion looks with more variety. For instance, after we found transition patterns, some morphing frames would be generated. These frames are not belong to the original motion, but by taking them into considerations in frame-by-frame comparison during real-time processing, we could create more different motion sequences.

\section{References}

Schödl, A., Szeliski, R., SAlesin, D. H., AND Essa, I. 2000. Video textures. In SIGGRAPH 2000, 489-498. 